\title{
LOCAL SMOOTH SOLUTIONS OF THE INCOMPRESSIBLE $K-\varepsilon$ MODEL AND THE LOW TURBULENT DIFFUSION LIMIT*
}

\author{
JULIEN MATHIAUD ${ }^{\dagger}$
}

\begin{abstract}
The aim of this paper is to study the local in time well posedness of the incompressible $k-\varepsilon$ model in a $3 \mathrm{~d}$ periodic domain. In the case when turbulent diffusion is much smaller than dissipation, asymptotic expansions are also derived, supposing that the velocity of the fluid can be neglected.
\end{abstract}

Key words. turbulence, $k-\varepsilon$, low turbulent diffusion limit

\section{Introduction}

The $k-\varepsilon$ model is widely used in various physical models to assess isotropic turbulence effects (see [19]). It is based on two scalar quantities characterizing turbulence: the kinetic turbulent energy and the rate of dissipation of turbulent energy. This model, proposed by Launder and Spalding ([12]), was designed to model the evolution of large turbulent structures and their effect on the large scale mean flow. Its main applications can be found in aerodynamics, for instance to study the influence of turbulence on airfoil boundary layers (cf. [4] and [7]). It is also considered for modelling turbulent mixing induced by Rayleigh-Taylor, Kelvin-Helmholtz and Richtmyer-Meshkov instabilities, for instance in an astrophysical context.

As a matter of fact, observations of the famous supernova 1987A have indicated that radioactive cobalt is far more thoroughly distributed among the explosive debris in the envelope than was predicted by model calculations of thin-shell nucleosynthesis in the pre-supernova star. It suggests the occurrence of large-scale mixing in the ejecta during the explosion $[10,1]$. The most promising mechanism for explaining mixing in the ejecta is a combination of the Rayleigh-Taylor and Kelvin-Helmholtz instabilities. The Rayleigh-Taylor instability can arise in the supernova envelope when the outwardly moving shock wave from the initial explosion propagates through layers of the star with radial stratification of the heavy elements. As the shock passes through the composition interfaces (i.e., oxygen/silicon, helium/carbon+oxygen and hydrogen/helium), a rarefaction front moves back into the star, resulting in an effective reversal of gravity as low-density composition is pressure-accelerated into the underlying high-density composition. Any perturbation at the interface (i.e., velocity perturbation or spatial perturbation) will get amplified by the Rayleigh-Taylor and Richtmyer-Meshkov instabilities and result in the overturning of light and heavy elements. This results in the mixing of heavy elements throughout the envelope of the supernova remnant, with associated observational consequences in the light curve. A further mixing will occur as the dense "tongues" of the heavy elements experience differential shear with the lighter elements, resulting in Kelvin-Helmholtz instabilities. Thus the fingers of heavy and light fluid that developed initially get far more distorted and the mixing layer increases its width. Eventually these instabilities become so nonlinear that the mixing layer appears to become fully turbulent. The properties of turbulently mixed layers may be equally important in understanding how interstellar clouds get reprocessed back into the interstellar medium. Efficient mixing of

\footnotetext{
*Received: April 2, 2007; accepted (in revised version): March 9, 2008. Communicated by Francois Golse.

${ }^{\dagger}$ CEA/DIF, B.P. 12, 91680 Bruyères le Châtel, France, \& E.N.S. Cachan, CMLA, 61 avenue du président Wilson, 94230 Cachan cedex, France (mathiaud@cmla.ens-cachan.fr).
} 
cloud and inter-cloud matter has been shown to occur after clouds get crushed by the interaction of strong shocks from supernova remnants [8] [9].

Here we study the mathematical properties of the incompressible model. Let us emphasize that this model is widely used in industrial codes because of its physical relevance and its simplicity. In order to introduce the mathematical setting and write the model equations, let us first introduce some notations. The domain will be a 3dimensional box $\mathbb{T}^{3}=\mathbb{R}^{3} /(2 \pi \mathbb{Z})^{3}$ with periodic boundary conditions in order to avoid additional difficulties (specific physical modelling and mathematical tools are needed to handle boundaries but here we choose not to deal with boundary layers for sake of simplicity). The system of equations can be written as follows (cf.[19]):

$$
\begin{gathered}
\frac{\partial U}{\partial t}+U \cdot \nabla U+\nabla P-\nu \Delta U-\nabla \cdot R=0, \\
\nabla \cdot U=0, \\
\frac{\partial k}{\partial t}+U \cdot \nabla k-\frac{c_{\mu}}{2} \frac{k^{2}}{\varepsilon}\left|\nabla U+\nabla U^{T}\right|^{2}-\nabla \cdot\left(c_{\mu} \frac{k^{2}}{\varepsilon} \nabla k\right)+\varepsilon=0, \\
\frac{\partial \varepsilon}{\partial t}+U \cdot \nabla \varepsilon-\frac{c_{1}}{2} k\left|\nabla U+\nabla U^{T}\right|^{2}-\nabla \cdot\left(c_{\varepsilon} \frac{k^{2}}{\varepsilon} \nabla \varepsilon\right)+c_{2} \frac{\varepsilon^{2}}{k}=0, \\
U(0, x)=U^{0}(x), \quad k(0, x)=k^{0}(x), \quad \varepsilon(0, x)=\varepsilon^{0}(x),
\end{gathered}
$$

where $U:=U(t, x) \in \mathbb{R}^{3}$ denotes the large scale flow, $k:=k(t, x)$ the kinetic turbulent energy, $\varepsilon:=\varepsilon(t, x)$ its dissipation rate. $P=P(t, x)$ stands for the mean pressure of the fluid; as usual in incompressible fluid models, it may be interpreted as a lagrangian multiplier of the constraint (1.2). Moreover, $R:=R(t, x)$ denotes the Reynolds stress tensor, given by

$$
R=-\frac{2}{3} k I+c_{\mu} \frac{k^{2}}{\varepsilon}\left(\nabla U+\nabla U^{T}\right) .
$$

Finally, $\nu$ denotes the constant positive molecular viscosity of the fluid, while $c_{1}, c_{2}$, $c_{\mu}$ and $c_{\varepsilon}$ are given positive constants that allow to capture the large scale features of turbulence (typical numerical values taken in realistic computations are: $c_{1}=0.126$, $c_{2}=1.92, c_{\mu}=0.09$ and $\left.c_{\varepsilon}=0.07\right)$.

For a survey about uniqueness and existence results concerning the Navier-Stokes equation without the $k-\varepsilon$ extension, we refer to [16]. Some inequalities on $k$ and $\varepsilon$ can be found in [14] and [19]: they are extended here using the same ideas. There also exist some results on a modified $k-\varepsilon$ model (the so-called $\phi-\theta$ model) given by Mohammadi and Lewandowski ([15]) when $U$ is supposed to be known so that one has only to solve the equations on $\phi$ and $\theta$ (which are very close to (1.3) and (1.4)); nonetheless the solutions which are found are more general than the ones found here - in a weaker sense - and still unique. More recently, the elliptic problem associated to $k$ and $\varepsilon$ has been studied $([6,13])$ : this problem arises in geophysics when one intends to study stationary mean flows. Weak solutions have been found: the main difficulty is to deal with the control of the singularity of the turbulent viscosity $c_{\mu} \frac{k^{2}}{\varepsilon}$, when $k$ and $\varepsilon$ both tend to zero, as we will also see in this paper.

As far as we know, the following is the first result on smooth solutions for the coupled equations (1.1)-(1.6).

The aim of this article is to provide a first study of this problem:

1. First we prove the following result for short enough time: 
THEOREM 1.1 (existence and uniqueness of smooth solutions).

The following two results hold:

(a) Let the initial data $U^{0}, k^{0}, \varepsilon^{0}$ be in $H^{s}$ for $s>4+3 / 2(s \in \mathbb{N})$ with $k^{0}$ and $\varepsilon^{0}$ bounded away from zero by a positive constant. Then there exists a positive $T$ and a strong ${ }^{1}$ solution $(U, k, \varepsilon)$ to system (1.1)-(1.6) on $[0, T]$ which belongs to $\mathcal{C}\left([0, T] ; H^{s}\left(\mathbb{T}^{3}\right)\right) \cap \mathcal{C}^{1}\left([0, T] ; H^{s-2}\left(\mathbb{T}^{3}\right)\right)$ such that $k$ and $\varepsilon$ remain positive on $[0, T]$.

(b) Moreover let $\left(U_{1}, k_{1}, \varepsilon_{1}\right)$ and $\left(U_{2}, k_{2}, \varepsilon_{2}\right)$ be two solutions of system (1.1)(1.6) in the sense of distributions. We suppose that they belong to $\mathcal{C}\left([0, T] ; H^{2}\left(\mathbb{T}^{3}\right)\right) \cap \mathcal{C}^{1}\left([0, T] ; L^{2}\left(\mathbb{T}^{3}\right)\right)$ and that $k_{1}, \varepsilon_{1}, k_{2}$ and $\varepsilon_{2}$ are positive functions.

If $U_{1}^{0}=U_{2}^{0}, k_{1}^{0}=k_{2}^{0}, \varepsilon_{1}^{0}=\varepsilon_{2}^{0}$, then $U_{1}=U_{2}, k_{1}=k_{2}, \varepsilon_{1}=\varepsilon_{2}$ on $[0, T]$.

2. Then we study a particular regime when turbulent diffusion effects are small compared with dissipation and when the mean flow is supposed to be at rest (so that $U$ is considered to be identically 0 ). Rescaling the $k-\varepsilon$ system is classical in order to obtain further information. For instance, S. Lasserre provides a study of the system depending on the pair of variables $\left(k, \ln \left(k^{\sigma k / \sigma \varepsilon} / \varepsilon\right)\right)$ ([11]) to study compact solutions, but here we want to stay as close as possible to the original equations to preserve the parabolic behavior and also to be in accordance with physical data. We consider the following non-dimensional system (see the beginning of Sec. 4):

$$
\begin{aligned}
& \frac{\partial k}{\partial t}-\eta \nabla \cdot\left(\frac{k^{2}}{\varepsilon} \nabla k\right)+A \varepsilon=0, \\
& \frac{\partial \varepsilon}{\partial t}-\eta \nabla \cdot\left(\frac{c_{\varepsilon}}{c_{\mu}} \frac{k^{2}}{\varepsilon} \nabla \varepsilon\right)+c_{2} A \frac{\varepsilon^{2}}{k}=0,
\end{aligned}
$$

with $A=\varepsilon^{0} T / k^{0}, \eta=c_{\mu}\left(k^{0}\right)^{2} T /\left(\varepsilon^{0} L^{2}\right), k^{0}$ denoting the typical kinetic turbulent energy, $\varepsilon^{0}$ the rate of kinetic turbulent energy dissipation, $T$ and $L$ the typical time and length scales of the physical situation.

We make an asymptotic expansion with respect to $\eta$ for this model since for some typical physical set of values, $\eta$ is negligible while $A$ 's value is of order 1 . The difference between the solution $(k, \varepsilon)$ of equations (1.7)-(1.8) and the first terms of its $\eta$ expansion $\left(k_{0}+\eta k_{1}, \varepsilon_{0}+\eta \varepsilon_{1}\right)$ can be controlled through the following result:

Theorem 1.2 (Asymptotic expansion). Let $k^{0}$, $\varepsilon^{0}$ belong to $\left.H^{7}\left(\mathbb{T}^{3}\right)\right)$ and be bounded away from zero by positive constants, and let $k$ and $\varepsilon$ be positive solutions of (1.7) and (1.8) bounded away from zero in $C^{1}\left([0, T] ; H^{5}\left(\mathbb{T}^{3}\right)\right)$. Then there exists a positive time $T^{\prime} \leq T$ and a constant $C$ such that for all $t \leq T^{\prime}$

$$
\left\|k-k_{0}-\eta k_{1}\right\|_{L^{\infty}\left(\mathbb{T}^{3}\right)}(t) \leq C \eta^{\frac{3}{2}}, \quad\left\|\varepsilon-\varepsilon_{0}-\eta \varepsilon_{1}\right\|_{L^{\infty}\left(\mathbb{T}^{3}\right)}(t) \leq C \eta^{\frac{3}{2}},
$$

where $k_{0}, k_{1}, \varepsilon_{0}, \varepsilon_{1}$ are solutions of the following systems of ordinary differential and partial differential equations:

1. Zeroth order system

$$
\begin{gathered}
\frac{\partial k_{0}}{\partial t}+A \varepsilon_{0}=0, \quad k_{0}(0, .)=k^{0}(.), \\
\frac{\partial \varepsilon_{0}}{\partial t}+c_{2} A \frac{\varepsilon_{0}^{2}}{k_{0}}=0, \quad \varepsilon_{0}(0, .)=\varepsilon^{0}(.) .
\end{gathered}
$$

\footnotetext{
${ }^{1}$ By strong we mean a classical $\mathcal{C}_{t}^{1}\left(C_{x}^{2}\right)$ solution
} 
2. First order system

$$
\begin{aligned}
& \frac{\partial k_{1}}{\partial t}-\nabla \cdot\left(\frac{k_{0}^{2}}{\varepsilon_{0}} \nabla k_{0}\right)+A \varepsilon_{1}=0, \quad k_{1}(0, .)=0 \\
& \frac{\partial \varepsilon_{1}}{\partial t}-\nabla \cdot\left(\frac{c_{\varepsilon}}{c_{\mu}} \frac{k_{0}^{2}}{\varepsilon_{0}} \nabla \varepsilon_{0}\right)+c_{2} A\left(\frac{2 \varepsilon_{0} \varepsilon_{1}}{k_{0}}-\frac{\varepsilon_{0}^{2} k_{1}}{k_{0}^{2}}\right)=0, \quad \varepsilon_{1}(0, .)=0 .
\end{aligned}
$$

From a mathematical viewpoint, the main difficulty in the proof of Theorems 1 and 2 is the control of the positivity of $k$ and $\varepsilon$, since the mathematical model degenerates when $k$ or $\varepsilon$ vanish: because of the $\varepsilon$ (resp. $c_{2} \varepsilon^{2} / k$ ) term in Equ. (1.3) (resp.(1.4)), we cannot ensure strict positivity of $k$ (resp. $\varepsilon$ ).

Three classical mathematical tools are used all along the article to carry on the study. The first one is the maximum principle for parabolic PDE's. The second one is the use of energy methods for parabolic PDE's to obtain a priori estimates. The last one is the use of Sobolev embeddings (see [2] and [21]) and Gagliardo-Niremberg inequalities in order to control the different norms. Since we need smoothness of the solutions in order to be able to use these inequalities, our study is for data which belong to $H^{s}$ with $4+3 / 2$ so that the $L^{\infty}$-norms of the gradients of the data are bounded by the $H^{s}$-norms.

In Sec. 2 we give an a priori estimate and in Sec. 3 we solve the problem of existence and uniqueness of solutions of (1.1)-(1.6), proving the first theorem; in Sec. 4 we perform the expansion with respect to $\eta$ and prove the second theorem. All the results are given for small times, since there is no hope in controlling the strict positivity of $k$ and $\varepsilon$ for long times.

\section{Preliminary results}

We establish a priori estimates on $U, k$ and $\varepsilon$ solutions of system (1.1)-(1.6). In all the computations, we consider solutions $(U, k, \varepsilon)$ belonging to $C\left([0, T] ; H^{s}\left(\mathbb{T}^{3}\right)\right) \cap$ $C^{1}\left([0, T] ; H^{s-2}\left(\mathbb{T}^{3}\right)\right)$ for some $T>0$ and for any integer $s$ such that $s>4+3 / 2$ (and consequently belonging to $C^{2}\left([0, T] ; H^{s-4}\left(\mathbb{T}^{3}\right)\right)$ at least). All the integrals are computed on $\mathbb{T}^{3} ; k$ and $\varepsilon$ are supposed to be strictly positive quantities for $t \in[0, T]$. We systemically use Sobolev embedding $H^{s}\left(\mathbb{T}^{3}\right) \hookrightarrow \mathcal{C}^{s-2}\left(\mathbb{T}^{3}\right)$ so that the $(s-2)^{\text {th }}$ space derivatives of $U, k$ and $\varepsilon$ are $L^{\infty}$-bounded by the $H^{s}$ norm of $U, k$ and $\varepsilon$ (see [2]) in dimension 3 .

2.1. A priori estimates on the Navier-Stokes equations. We obtain the following estimates on smooth solutions of the incompressible Navier-Stokes equation:

Proposition 2.1 (Estimates on $U$ ). Let $U, k$ and $\varepsilon$ be solutions of (1.1)-(1.6) which belong to $C\left([0, T] ; H^{s}\left(\mathbb{T}^{3}\right)\right) \cap C^{1}\left([0, T] ; H^{s-2}\left(\mathbb{T}^{3}\right)\right)$, with $k$ and $\varepsilon$ strictly positive and $s \geq 4+3 / 2$. We have:

$$
\frac{d}{d t}\|U\|_{H^{s}}^{2} \leq C Q_{4 s+8}(U, k, \varepsilon)(t),
$$

where $C$ is a generic constant and $Q_{1}, \ldots, Q_{n}$ are functions defined by:

$$
\begin{aligned}
& Q_{1}(U, k, \varepsilon)(t)=1+\|U(t)\|_{H^{s}}+\|k(t)\|_{H^{s}}+\|\varepsilon(t)\|_{H^{s}}+\frac{1}{k_{\min }(t)}+\frac{1}{\varepsilon_{\min }(t)} \\
& Q_{n}=\left(Q_{1}\right)^{n}
\end{aligned}
$$


where we note

$$
\begin{array}{cl}
k_{\min }(t)=\min _{x \in \mathbb{T}^{3}} k(t, x), & k_{\max }(t)=\max _{x \in \mathbb{T}^{3}, 0 \leq s \leq t} k(s, x), \\
\varepsilon_{\min }(t)=\min _{x \in \mathbb{T}^{3}, 0 \leq s \leq t} \varepsilon(s, x), \quad \varepsilon_{\max }(t)=\max _{x \in \mathbb{T}^{3}, 0 \leq s \leq t}(\varepsilon(s, x)),
\end{array}
$$

and $D^{i} h$ denotes the $i^{\text {th }}$ derivative of a function $h$.

Proof. First note that is enough to prove,

$$
\frac{d}{d t}\left\|D^{s} U\right\|_{L^{2}}^{2} \leq C Q_{4 s+8}(U, k, \varepsilon)(t)
$$

since for any integer $s^{\prime}<s$ the result on $D^{s^{\prime}} U$ is easier to prove (Sobolev embeddings give better results, especially in the $L^{\infty}$-norm for lower order derivatives, so that we clearly have $\left.\left.\frac{d}{d t}\left\|D^{s^{\prime}} U\right\|_{L^{2}}^{2} \leq C Q_{4 s+8}(U, k, \varepsilon)(t)\right)\right)$.

From now on, $\alpha=\left(\alpha_{1}, \alpha_{2}, \alpha_{3}\right) \in \mathbb{N}^{3}$ denotes a multi-index of differentiation such that

$$
\partial^{\alpha}=\partial_{x_{1}}^{\alpha_{1}} \partial_{x_{2}}^{\alpha_{2}} \partial_{x_{3}}^{\alpha_{3}}, \quad|\alpha|=\sum_{i=1}^{3} \alpha_{i}
$$

denoting its length. $\beta \leq \alpha$ means that $\forall i, \beta_{i} \leq \alpha_{i}$, while $\beta+\gamma=\alpha$ means $\forall i, \beta_{i}+\gamma_{i}=$ $\alpha_{i}$. We first obtain estimates on $\partial^{\alpha} U(|\alpha|=s)$ : we differentiate the Navier-Stokes equations and take $\partial^{\alpha} U$ as test vector field.

$$
\frac{1}{2} \frac{d}{d t}\left\|\partial^{\alpha} U\right\|_{L^{2}}^{2}+\nu\left\|D^{1}\left(\partial^{\alpha} U\right)\right\|_{L^{2}}^{2}=(A)+(B)+(C)+(D)
$$

with:

$$
\begin{aligned}
& (A)=-b\left(U, \partial^{\alpha} U, \partial^{\alpha} U\right), \quad(B)=-b\left(\partial^{\alpha} U, U, \partial^{\alpha} U\right), \\
& (C)=-\sum_{\beta+\gamma=\alpha, s>|\beta|,|\gamma| \geq 1} b\left(\partial^{\beta} U, \partial^{\gamma} U, \partial^{\alpha} U\right), \\
& (D)=\int \partial^{\alpha} U \cdot\left(\nabla \cdot \partial^{\alpha}\left(\frac{k^{2}}{\varepsilon}\left(\nabla U+\nabla U^{T}\right)\right)\right) d x,
\end{aligned}
$$

where $b(u, v, w)$ is the trilinear form linked to the incompressible Navier-Stokes equation and defined on $\left(H^{1}\left(\mathbb{T}^{3}\right)\right)^{3}$ by

$$
b(u, v, w)=\sum_{1 \leq i, j \leq 3} \int u_{i} \partial_{i} v_{j} w_{j} d x
$$

(see [3] or [23] for notations and properties). Using $\nabla \cdot U=0$, one may note that the term in which pressure and $\nabla x \cdot(k I)$ appear vanished after integrating by parts. Moreover, (A) vanishes, since its last two arguments are equal and the first one is divergence free. (B) and (C) can be estimated by $C\|U\|_{H^{s}}^{3}$, thanks to Sobolev embeddings ( $C$ denotes a generic constant). For instance, for the $(C)$ term, either $\beta$ or $\gamma+1$ is less than $s-2(s \geq 5$ and $|\beta|+|\gamma|=s)$, so that $\partial^{\beta} U$ or $\partial_{i}\left(\partial^{\gamma} U\right)$ are $L^{\infty}$ bounded by $\|U\|_{H^{s}}$ (we recall that a function is continuous and its norm is controlled by its $H^{s}$ norm in the 3 -D torus as soon as $s>\frac{3}{2}$ : one can prove it using Fourier series, 
for instance $([21]))$, and since the two other terms of the trilinear form belong to $L^{2}$ at least, the trilinear form is well-defined and bounded by $C\|U\|_{H^{s}}^{3}$.

There remains the last term (D): integrating by parts and using the symmetry of $\nabla U+\nabla U^{T}$, one gets (there are no boundary terms, since we use periodic boundary conditions):

$$
\begin{aligned}
\int \partial^{\alpha} U \cdot\left(\nabla \cdot \partial^{\alpha}\right. & \left.\left(\frac{k^{2}}{\varepsilon}\left(\nabla U+\nabla U^{T}\right)\right)\right) d x \\
& =-\int \partial^{\alpha}\left(\frac{k^{2}}{\varepsilon}\left(\nabla U+\nabla U^{T}\right)\right): \partial^{\alpha} \frac{\nabla U+\nabla U^{T}}{2} d x .
\end{aligned}
$$

Then, studying the terms coming from $\partial^{\alpha}\left(\left(\nabla U+\nabla U^{T}\right) k^{2} / \varepsilon\right)$, we get three kinds of terms which satisfy the following inequalities (with $\beta+\gamma=\alpha, s \geq|\beta|,|\gamma| \geq 0$ ):

- when $\gamma=\alpha$ and $\beta=0$ :

$$
-\frac{1}{2} \int \frac{k^{2}}{\varepsilon} \partial^{\gamma}\left(\nabla U+\nabla U^{T}\right): \partial^{\alpha}\left(\nabla U+\nabla U^{T}\right) d x \leq 0
$$

- when $2 \leq|\gamma| \leq s-1$ :

Thanks to Young's inequality, one gets

$$
\begin{aligned}
& -\frac{1}{2} \int \partial^{\beta}\left(\frac{k^{2}}{\varepsilon}\right) \partial^{\gamma}\left(\nabla U+\nabla U^{T}\right): \partial^{\alpha}\left(\nabla U+\nabla U^{T}\right) d x \\
\leq & \frac{8}{\nu}\left(\left\|\partial^{\beta}\left(\frac{k^{2}}{\varepsilon}\right) \partial^{\gamma}\left(\nabla U+\nabla U^{T}\right)\right\|_{L^{2}}\right)^{2}+\frac{\nu}{8}\left\|\partial^{\alpha}\left(\nabla U+\nabla U^{T}\right)\right\|_{L^{2}}^{2} \\
\leq & \frac{8}{\nu}\left(\left\|\partial^{\beta}\left(\frac{k^{2}}{\varepsilon}\right) \partial^{\gamma}\left(\nabla U+\nabla U^{T}\right)\right\|_{L^{2}}\right)^{2}+\frac{\nu}{4}\left\|D^{1}\left(\partial^{\alpha} U\right)\right\|_{L^{2}}^{2} ;
\end{aligned}
$$

- when $|\gamma| \leq 1$ :

$$
\begin{aligned}
& -\frac{1}{2} \int \partial^{\beta}\left(\frac{k^{2}}{\varepsilon}\right) \partial^{\gamma}\left(\nabla U+\nabla U^{T}\right): \partial^{\alpha}\left(\nabla U+\nabla U^{T}\right) d x \\
\leq & \frac{\nu}{4}\left\|D^{1}\left(\partial^{\alpha} U\right)\right\|_{L^{2}}^{2}+\frac{8}{\nu}\left(\left\|\partial^{\beta}\left(\frac{k^{2}}{\varepsilon}\right) \partial^{\gamma}\left(\nabla U+\nabla U^{T}\right)\right\|_{L^{2}}\right)^{2} .
\end{aligned}
$$

In order to complete the proof, one has to note that in the last two cases, either $\partial^{\beta}\left(k^{2} / \varepsilon\right)$ or $\partial^{\gamma}\left(\nabla U+\nabla U^{T}\right)$ belong to $L^{\infty}\left(\mathbb{T}^{3}\right)$ because of the embedding $H^{2}\left(\mathbb{T}^{3}\right) \hookrightarrow$ $L^{\infty}\left(\mathbb{T}^{3}\right)$. Therefore, we obtain

$$
\begin{aligned}
& \frac{1}{2} \frac{d}{d t}\left\|\partial^{\alpha} U\right\|_{L^{2}}^{2}+\nu\left\|D^{1}\left(\partial^{\alpha} U\right)\right\|_{L^{2}}^{2} \\
\leq & \frac{\nu}{2}\left\|D^{1}\left(\partial^{\alpha} U\right)\right\|_{L^{2}}^{2}+C\left(\|U\|_{H^{s}}^{3}+\left\|\frac{k^{2}}{\varepsilon}\right\|_{H^{s}}^{2}\|U\|_{H^{s}}^{2}\right) .
\end{aligned}
$$

Differentiating $k^{2} / \varepsilon$, one gets after some computations that

$$
\left\|\frac{k^{2}}{\varepsilon}\right\|_{H^{s}} \leq C\left(\|k(t)\|_{H^{s}}+\|\varepsilon(t)\|_{H^{s}}+\frac{1}{k_{\min }(t)}+\frac{1}{\varepsilon_{\min }(t)}\right)^{2 s+3}
$$

since $1 / \varepsilon$ is in $H^{s}$ (because $\varepsilon$ is in $H^{s}$ and bounded below by a strictly positive constant so that $1 / \varepsilon$ in $L^{\infty}$ and consequently in $L$ - the Torus is bounded) and $H^{s}$ 
is an algebra. Using the definition of $Q_{n}$, we get a bound for $\partial^{\alpha} U$ :

$$
\frac{d}{d t}\left\|\partial^{\alpha} U\right\|_{L^{2}}^{2} \leq C Q_{4 s+8}(U, k, \varepsilon)(t)
$$

This result holds for all the derivatives, so this completes the proof.

2.2. A priori estimates on $k$ and $\varepsilon$. We are now able to control positive lower bounds $k$ and $\varepsilon$ through the following lemma.

LEMMA 2.1 (Maximum principle). Let $U, k$ and $\varepsilon$ be solutions of System (1.1)(1.6) which belong to $C\left([0, T] ; H^{s}\left(\mathbb{T}^{3}\right)\right) \cap C^{1}\left([0, T] ; H^{s-2}\left(\mathbb{T}^{3}\right)\right)$, with $k$ and $\varepsilon$ bounded below by a strictly positive constant. Let $k^{0}$, $\varepsilon^{0}$ belong to $H^{s}\left(\mathbb{T}^{3}\right)$ ) and be bounded away from zero by strictly positive constants. We have for all $t \geq 0$ :

$$
\begin{aligned}
& \frac{1}{k_{\min }(t)}-\frac{1}{k_{\min }(0)} \leq C \frac{t}{k_{\text {min }}^{2}(t)} \sup _{0 \leq t^{\prime} \leq t}\left\|\varepsilon\left(t^{\prime}, .\right)\right\|_{H^{s}}, \\
& \frac{1}{\varepsilon_{\min }(t)}-\frac{1}{\varepsilon_{\min }(0)} \leq C \frac{t}{k_{\min }(t) \varepsilon_{\min }^{2}(t)} \sup _{0 \leq t^{\prime} \leq t}\left\|\varepsilon\left(t^{\prime}, .\right)\right\|_{H^{s}}^{2} .
\end{aligned}
$$

Proof. We only prove the result for $k_{\min }$. One has to note that $k$ belongs to $C^{2}\left([0, T] ; H^{2}\left(\mathbb{T}^{3}\right)\right)$ and consequently to $C^{2}\left([0, T] ; \mathcal{C}\left(\mathbb{T}^{3}\right)\right)$ since $k$ is a solution of the problem so that $k_{\text {min }}$ is continuous. For all $t \leq T, \delta>0$ and $u \leq t$ we define the following function,

$$
r_{\delta}(u, x)=\delta(1+u)+C \frac{u}{k_{\min }^{2}(t)} \sup _{0 \leq t^{\prime} \leq t}\left\|\varepsilon\left(t^{\prime}, .\right)\right\|_{H^{s}}-\frac{1}{k(u, x)}+\frac{1}{k_{\min }(0)},
$$

which belongs to $C^{1}\left([0, t) ; H^{s-2}\left(\mathbb{T}^{3}\right)\right)$, where $C$ is the Sobolev constant of the embedding $H^{s}\left(\mathbb{T}^{3}\right) \hookrightarrow L^{\infty}\left(\mathbb{T}^{3}\right)(s>3 / 2)$. One has to note that, thanks to the definition of $k_{\min }$,

$$
\forall x \in \mathbb{T}^{3}, \quad r_{\delta}(0, x) \geq \delta>0 .
$$

Let us suppose that $r_{\delta}$ vanishes at some point in $(0, t) \times \mathbb{T}^{3}$ and let us denote by $t_{1}$ the first time such that there exists $x_{1} \in \mathbb{T}^{3}$ satisfying $r_{\delta}\left(t_{1}, x_{1}\right)=0$ (note that $t_{1}$ is uniquely defined, whereas $x_{1}$ may not be unique).

Since $0<t_{1}<t, \partial_{t} k^{-1}\left(t_{1}, x_{1}\right)$ is well defined. Moreover, thanks to the definition of $t_{1}$, we get that $\left(t_{1}, x_{1}\right)$ is a local minimum in space of $r_{\delta}$ and consequently a minimum of $x \mapsto-k^{-1}\left(t_{1}, x\right)$ and a minimum of $k$. As a result, $\nabla k\left(t_{1}, x_{1}\right)=0$ and $\Delta k\left(t_{1}, x_{1}\right) \geq 0$ $\left(\mathbb{T}^{3}\right.$ is an open set). Hence the $k$ equation at point $\left(t_{1}, x_{1}\right)$ gives:

$$
\frac{\partial k}{\partial t}\left(t_{1}, x_{1}\right) \geq-\varepsilon\left(t_{1}, x_{1}\right) \text {. }
$$

Using Sobolev embeddings, one gets for all the minima at time $t_{1}$

$$
\frac{\partial k}{\partial t}\left(t_{1}, x_{1}\right) \geq-C\left\|\varepsilon\left(t_{1}, .\right)\right\|_{H^{s}}, \quad \text { so that } \frac{\partial}{\partial t}\left(\frac{1}{k}\right)\left(t_{1}, x_{1}\right) \leq C \frac{\left\|\varepsilon\left(t_{1}, .\right)\right\|_{H^{s}}}{k_{\min }\left(t_{1}\right)^{2}}
$$

Finally, we get

$$
\begin{aligned}
\frac{\partial}{\partial t} r_{\delta}\left(t_{1}, x_{1}\right) & \geq \delta+C \frac{1}{k_{\min }^{2}(t)} \sup _{0 \leq t^{\prime} \leq t}\left\|\varepsilon\left(t^{\prime}, .\right)\right\|_{H^{s}}-C \frac{\left\|\varepsilon\left(t_{1}, .\right)\right\|_{H^{s}}}{k_{\min }\left(t_{1}\right)^{2}} \\
& \geq \delta+0\left(\text { thanks to the definition of } k_{\min }\right) \\
& >0 .
\end{aligned}
$$


Therefore, the function $u \mapsto r_{\delta}\left(u, x_{1}\right)$ is negative for $u<t_{1}$ in the neighborhood of $t_{1}$. $t_{1}$ is the first time for which $r_{\delta}$ vanishes and $r_{\delta}$ is necessarily positive on $] 0, t_{1}\left[\times \mathbb{T}^{3}\right.$ by continuity, we obtain a contradiction and as a consequence $r_{\delta}$ is a strictly positive function on $\left[0, t\left[\times \mathbb{T}^{3}\right.\right.$ and a positive function by continuity at time $t$ for all $x$. Eventually, letting $\delta$ tend to zero by continuity, we get:

$$
\begin{aligned}
\frac{1}{k(u, x)} & \leq C \frac{u}{k_{\min }^{2}(t)} \sup _{0 \leq t^{\prime} \leq t}\left\|\varepsilon\left(t^{\prime}, .\right)\right\|_{H^{s}}+\frac{1}{k_{\min }(0)} \\
& \leq C \frac{t}{k_{\min }^{2}(t)} \sup _{0 \leq t^{\prime} \leq t}\left\|\varepsilon\left(t^{\prime}, .\right)\right\|_{H^{s}}+\frac{1}{k_{\min }(0)}
\end{aligned}
$$

(thanks to the definition of $k_{\min }$ ). Taking the maximum in space and time, one gets the result.

In addition, we obtain the following estimates for $k$ and $\varepsilon$ (strict positivity of both $k$ and $\varepsilon$ is necessary for the inequalities).

Proposition 2.2 (a priori estimates on $k$ and $\varepsilon$ ). Let $U, k$ and $\varepsilon$ be solutions of (1.1)-(1.6) which belong to $C\left([0, T] ; H^{s}\left(\mathbb{T}^{3}\right)\right) \cap C^{1}\left([0, T] ; H^{s-2}\left(\mathbb{T}^{3}\right)\right)$, with $k$ and $\varepsilon$ strictly positive and $s \geq 4+3 / 2$. We have:

$$
\frac{d}{d t}\|k\|_{H^{s}}^{2} \leq C Q_{4 s+9}(U, k, \varepsilon), \quad \frac{d}{d t}\|\varepsilon\|_{H^{s}}^{2} \leq C Q_{4 s+9}(U, k, \varepsilon) .
$$

Proof. We only give a proof of the second inequality (the same tools are used to obtain the first result). In order to prove it, we only prove that

$$
\frac{d}{d t}\left\|D^{s} \varepsilon\right\|_{L^{2}}^{2} \leq C Q_{4 s+9}(U, k, \varepsilon)
$$

since for lower order derivatives the same inequality is easier to prove (Sobolev embeddings give better results especially in the $L^{\infty}$-norm for lower order derivatives). Differentiating the $\varepsilon$ equation $s$ times in the direction $\alpha \in \mathbb{N}^{3}$, multiplying by $\partial^{\alpha} \varepsilon$, and integrating by parts the diffusive term, one gets:

$$
\frac{1}{2} \frac{d}{d t}\left\|\partial^{\alpha} \varepsilon\right\|_{L^{2}}^{2}+(A)+(B)+(C)+(D)=0
$$

with:

$$
\begin{aligned}
& (A)=\int \partial^{\alpha}(U \cdot \nabla \varepsilon)\left(\partial^{\alpha} \varepsilon\right) d x, \\
& (B)=-\int \frac{c_{1}}{2} \partial^{\alpha}\left(k\left|\nabla U+\nabla U^{T}\right|^{2}\right)\left(\partial^{\alpha} \varepsilon\right) d x, \\
& (C)=\int\left(\partial^{\alpha} \cdot \nabla \varepsilon\right) \partial^{\alpha}\left(c_{\mu} \frac{k^{2}}{\varepsilon} \nabla \varepsilon\right) d x, \\
& (D)=\int \partial^{\alpha} \varepsilon \partial^{\alpha}\left(\frac{\varepsilon^{2}}{k}\right) d x .
\end{aligned}
$$

The $(A)$ term can be treated like the trilinear term of the Navier-Stokes equation using that $U$ is divergence free,

$$
\int\left(U \cdot\left(\partial^{\alpha} \nabla \varepsilon\right)\right)\left(\partial^{\alpha} \varepsilon\right) d x=0
$$


so that all the other terms of $A$ contain derivatives of $\varepsilon$ of order less than $s$, which, using Sobolev embedding, leads to $|(A)| \leq C\|U\|_{H^{s}}\|\varepsilon\|_{H^{s}}^{2}$.

By integrating by parts the $(B)$ term, one gets that

$$
|(B)| \leq C\|\varepsilon\|_{H^{s+1}}|| k\left|\nabla U+\nabla U^{T}\right|^{2} \|_{H^{s-1}} .
$$

Using that $H^{s}$ is an algebra since $s>3 / 2$, we finally obtain:

$$
|(B)| \leq C|| D^{s+1} \varepsilon\left\|_{L^{2}}\right\| k\left\|_{H^{s}}\right\| U \|_{H^{s}}^{2} .
$$

After some computations one obtains the following result on $(D)$ :

$$
|(D)| \leq C\left(\|\varepsilon\|_{H^{s}}+\|k\|_{H^{s}}\right)^{2 s+4} \leq C Q_{2 s+4}(U, k, \varepsilon)(T) .
$$

Finally, there remains to study the diffusive term $(C)$ whose most important term satisfies (using the positivity of $k^{2} / \varepsilon$ ):

$$
\int \partial^{\alpha} \nabla \varepsilon \cdot\left(c_{\mu} \frac{k^{2}}{\varepsilon} \partial^{\alpha}(\nabla \varepsilon)\right) d x \geq c_{\mu} \frac{k_{m i n}^{2}(t)}{\|\varepsilon(t, .)\|_{H^{s}}}\left\|\partial^{\alpha}(\nabla \varepsilon)\right\|_{L^{2}}^{2} .
$$

The other terms of $(C)$ are bounded by

$$
\frac{1}{2} r c_{\mu} \frac{k_{\text {min }}^{2}(t)}{\|\varepsilon\|_{H^{s}}}\left\|\partial^{\alpha}(\nabla \varepsilon)\right\|_{L^{2}}^{2}+\frac{1}{r c_{\mu}} 2 \frac{\|\varepsilon\|_{H^{s}}}{k_{\text {min }}^{2}(t)}\left\|\frac{k^{2}}{\varepsilon}\right\| H_{H^{s}}^{2}\|\varepsilon\|_{H^{s}}^{2},
$$

Using Young's inequality and Sobolev embeddings for all $r>0$.

Since we already know that

$$
\left\|\frac{k^{2}}{\varepsilon}(t, .)\right\|_{H^{s}} \leq C\left(\|k(t)\|_{H^{s}}+\|\varepsilon(t)\|_{H^{s}}+\frac{1}{k_{\min }(t)}+\frac{1}{\varepsilon_{\min }(t)}\right)^{2 s+3},
$$

we obtain for another constant $\mathrm{C}$ depending only on the number of terms coming from (C) (choosing a good $r$ to eliminate the highest derivatives):

$$
(C) \geq \frac{1}{2} \frac{k_{\min }^{2}(t)}{\|\varepsilon(t, .)\|_{H^{s}}}\left\|D^{s+1} \varepsilon\right\|_{L^{2}}^{2}-C Q_{4 s+9}(U, k, \varepsilon)(t) .
$$

Combining the results for $(A),(B),(C)$ and $(D)$ one gets:

$$
\frac{1}{2} \frac{d}{d t}\left\|\partial^{\alpha} \varepsilon\right\|_{L^{2}}^{2} \leq C Q_{4 s+9}(U, k, \varepsilon)(t)
$$

(Note that the $(B)$ term is controlled by the $(C)$ term, using again $a b \leq a^{2}+b^{2}$.)

2.3. Positive lower bounds for $k$ and $\varepsilon$. We establish an a priori estimate for the turbulent fields $k$ and $\varepsilon$ and then prove that solutions of the system can be controlled locally in time.

Proposition 2.3. Let $s \in \mathbb{N}$ such that $s \geq 4+3 / 2$ and $U, k$ and $\varepsilon$ be smooth solutions of (1.1)-(1.6) in $C\left([0, T] ; H^{s}\left(\mathbb{T}^{3}\right)\right) \cap C^{1}\left([0, T] ; H^{s-2}\left(\mathbb{T}^{3}\right)\right)$. Let $k^{0}$, $\varepsilon^{0}$ belong to $\left.H^{s}\left(\mathbb{T}^{3}\right)\right)$ and be bounded away from zero by strictly positive constants. Then there exists a positive time $T^{\prime}\left(T^{\prime} \leq T\right)$ such that $k$, $\varepsilon$ remain strictly positive on $\left[0, T^{\prime}\right]$ (bounded 
below by a strictly positive constant) and such that $U, k$ and $\varepsilon$ have finite $H^{s}$ norm.

Proof. Thanks to the previous results we obtain

$$
\frac{d}{d t}\left(\|\varepsilon\|_{H^{s}}^{2}+\|k\|_{H^{s}}^{2}+\|U\|_{H^{s}}^{2}\right) \leq C Q_{4 s+9}(U, k, \varepsilon)
$$

so that

$$
\begin{aligned}
\|\varepsilon\|_{H^{s}}^{2}(t)+\|k\|_{H^{s}}^{2}(t)+\|U\|_{H^{s}}^{2}(t) \leq & \|\varepsilon\|_{H^{3}}^{2}(0)+\|k\|_{H^{3}}^{2}(0)+\|U\|_{H^{3}}^{2}(0) \\
& +C \int 0^{t} Q_{4 s+9}(U, k, \varepsilon)(s) d s
\end{aligned}
$$

We introduce the following continuous increasing and positive function $f$,

$$
\begin{aligned}
f(t)= & \left.1+\sup _{0 \leq t^{\prime} \leq t} \| k\left(t^{\prime}, .\right)\right)\left\|_{H^{s}}^{2}+\sup _{0 \leq t^{\prime} \leq t}\right\| \varepsilon\left(t^{\prime}, .\right)\left\|_{H^{s}}^{2}+\sup _{0 \leq t^{\prime} \leq t}\right\| U\left(t^{\prime}\right) \|_{H^{s}}^{2} \\
& +\frac{1}{k_{\min }(t)}+\frac{1}{\varepsilon_{\min }(t)} .
\end{aligned}
$$

Using (2.2) and the maximum principle to control $\frac{1}{k_{\min }(t)}+\frac{1}{\varepsilon_{\min }(t)}$, one gets:

$$
\begin{aligned}
f(t) \leq f(0)+C\left(\frac{t}{k_{\text {min }}^{2}(t)} \sup _{0 \leq u \leq t}\|\varepsilon(u, .)\|_{H^{s}}\right. \\
\left.\quad+\frac{t}{k_{\min }(t) \varepsilon_{\text {min }}^{2}(t)} \sup _{0 \leq u \leq t}\|\varepsilon(u, .)\|_{H^{s}}^{2}+\int_{0}^{t} f^{4 s+9}\right) \\
\leq f(0)+3 C t f^{4 s+9}(t),
\end{aligned}
$$

because

- $\forall t^{\prime} \leq t$

$$
\begin{aligned}
& \left\|k\left(t^{\prime}\right)\right\|_{H^{s}}+\left\|\varepsilon\left(t^{\prime}\right)\right\|_{H^{s}}+\left\|U\left(t^{\prime}\right)\right\|_{H^{s}} \\
& \quad \leq 3\left(1+\sup _{0 \leq u \leq t}\|k(u)\|_{H^{s}}^{2}+\sup _{0 \leq u \leq t}\|\varepsilon(u)\|_{H^{s}}^{2}+\sup _{0 \leq u \leq t}\|U(u)\|_{H^{s}}^{2}\right),
\end{aligned}
$$

- $\forall t, \frac{1}{k_{\min }(t)}-\frac{1}{k_{\min }(0)} \leq C \frac{t}{k_{\min }^{2}(t)} \sup _{0 \leq u \leq t}\|\varepsilon(u, .)\|_{H^{s}}$,

- $\forall t, \frac{1}{\varepsilon_{\min }(t)}-\frac{1}{\varepsilon_{\min }(0)} \leq C \frac{t}{k_{\min }(t) \varepsilon_{\min }^{2}(t)} \sup _{0 \leq u \leq t}\|\varepsilon(u, .)\|_{H^{s}}^{2}$.

If we denote by $T^{\prime}(M)$ the first time $f(t)$ is equal to $M$, we obtain that $T^{\prime}(M) \geq(M-f(0)) /\left(3 C M^{4 s+9}\right)$. Then, studying the ratio $(M-f(0))\left(3 C M^{4 s+9}\right)$, one concludes that $f$ cannot blow up on $\left[0, T^{\prime}\right]$ with $T^{\prime}$ defined by $T^{\prime}=$ $\min \left(T, 1 /\left(24 C f(0)^{4 s+9}\right)\right)(k, \varepsilon$ and $U$ are defined on $[0, T])$. This completes the proof of the proposition. 


\section{Existence and uniqueness of solutions for the whole system}

Thanks to the ideas used to obtain the a priori estimates, we are able to prove Theorem 1.1.

3.1. Existence. We only give a sketch of proof for the existence. We use an iterative method to obtain the result. We denote by $U^{n}, k^{n}$ and $\varepsilon^{n}\left(k^{n}\right.$ and $\varepsilon^{n}$ are supposed to be strictly positive) the $n$-th iterate of $U, k$ and $\varepsilon$ defined on $\left[0, t_{n}\right]$ : the time of existence depends on $n$; we have to prove that it can be bounded below. $U^{0}$, $k^{0}$ and $\varepsilon^{0}$ are the initial data defined above.

3.1.1. Iterative process. We obtain $U^{n+1}, k^{n+1}$ and $\varepsilon^{n+1}$ through the following iterative process:

$$
\begin{aligned}
& \frac{\partial U^{n+1}}{\partial t}+U^{n} \cdot \nabla U^{n+1}+\nabla P^{n+1}-\nu \Delta U^{n+1} \\
&= \nabla \cdot\left(-\frac{2}{3} k^{n} I+c_{\mu} \frac{\left(k^{n}\right)^{2}}{\varepsilon^{n}}\left(\nabla U^{n+1}+\nabla U^{n+1^{T}}\right)\right), \\
& \nabla \cdot U^{n+1}=0, \\
&=\frac{\partial k^{n+1}}{\partial t}+U^{n+1} \cdot \nabla k^{n+1}-\nabla \cdot\left(c_{\mu} \frac{\left(k^{n}\right)^{2}}{\varepsilon^{n}} \nabla k^{n+1}\right) \\
& \frac{c_{\mu}}{\varepsilon^{n}}\left|\nabla U^{n+1}+\nabla U^{n+1}\right|^{2}-\varepsilon^{n}, \\
& \frac{\partial \varepsilon^{n+1}}{\partial t}+U^{n+1} \cdot \nabla \varepsilon^{n+1}-\nabla \cdot\left(c_{\varepsilon} \frac{\left(k^{n}\right)^{2}}{\varepsilon^{n}} \nabla \varepsilon^{n+1}\right) \\
&=\frac{c_{1}}{2} k^{n}\left|\nabla U^{n+1}+\nabla U^{n+1}\right|^{2}-c_{2} \frac{\left(\varepsilon^{n}\right)^{2}}{k^{n}}, \\
& U^{n+1}(0, x)=U^{0}(x), \\
& k^{n+1}(0, x)=k^{0}(x), \\
& \varepsilon^{n+1}(0, x)=\varepsilon^{0}(x) .
\end{aligned}
$$

3.1.2. Solving the iterative scheme. To solve the iterative scheme, we need to solve the following equations:

1. The first one is a linear parabolic equation with a Lagrange multiplier $(P)$ :

$$
\begin{aligned}
\frac{\partial U}{\partial t}+b \cdot \nabla U+\nabla P-\nu \Delta U & =\nabla \cdot\left(a\left(\nabla U+\nabla U^{T}\right)\right), \\
\nabla \cdot U & =0 \\
U(0, x) & =U^{0}(x),
\end{aligned}
$$

with $a, b$ in $\mathcal{C}\left([0, T] ; H^{s}\left(\mathbb{T}^{3}\right)\right) \cap \mathcal{C}^{1}\left([0, T] ; H^{s-2}\left(\mathbb{T}^{3}\right)\right)$ and $a$ strictly positive (we omit the term $\nabla \cdot\left(-2 k^{n} I / 3\right)$, since it can be considered a part of the pressure). This equation has a unique solution in $\mathcal{C}\left([0, T] ; H^{s}\left(\mathbb{T}^{3}\right)\right) \cap \mathcal{C}^{1}\left([0, T] ; H^{s-2}\left(\mathbb{T}^{3}\right)\right)$ for some $T$ depending on the data $a$ and $b$ (see [16] p. 69). 
Then for the solution of (3.1), (3.2) and (3.5), we obtain an $H^{s}$ estimate for all $s>3+3 / 2$ thanks to Sobolev embeddings and using the fact that $U$ is divergence free: there exists an integer $p(s)$ and a constant $C_{s, 0}$ which depend on $s$ such that:

$$
\begin{aligned}
& \frac{d}{d t}\left(\left\|U^{n+1}\right\|_{H^{s}}^{2}\right)+\frac{\nu}{2}\left\|D^{(s+1)} U^{n+1}\right\|_{L^{2}}^{2} \\
& \quad \leq C_{s, 0}\left(1+\left\|U^{n+1}\right\|_{H^{s}}^{2}+\left\|\varepsilon^{n}\right\|_{H^{s}}^{2}+\left\|k^{n}\right\|_{H^{s}}^{2}+\frac{1}{k_{\min }^{n}(t)}+\frac{1}{\varepsilon_{\min }^{n}(t)}\right)^{p(s)} .
\end{aligned}
$$

We keep a part of diffusion because $U^{n+1}$ appears in the equations of $k^{n+1}$ and $\varepsilon^{n+1}$.

2. The others are linear parabolic equations with variable diffusion coefficients:

$$
\begin{gathered}
\frac{\partial k}{\partial t}+b \cdot \nabla k-\nabla \cdot(a \nabla k)=c, \\
\frac{\partial \varepsilon}{\partial t}+b \cdot \nabla \varepsilon-\nabla \cdot\left(\frac{c_{\varepsilon}}{c_{\mu}} a \nabla \varepsilon\right)=d,
\end{gathered}
$$

with $a, b, c$ and $d$ in $\mathcal{C}\left([0, T] ; H^{s}\left(\mathbb{T}^{3}\right)\right) \cap \mathcal{C}^{1}\left([0, T] ; H^{s-2}\left(\mathbb{T}^{3}\right)\right), b$ strictly positive and $\nabla \cdot b=0$.

So we need to solve this parabolic system while conserving the strict positivity; the classic theory on parabolic systems give us a solution to the problem in $H^{s}$ (see [22]). Finally there exists an integer $q(s)$, a parameter $\mathbf{p}$ and a constant $C_{s, 1}$ which depend on $s$ such that:

$$
\begin{aligned}
\frac{d}{d t}\left\|k^{n+1}\right\|_{H^{s}}^{2} \leq C_{s, 1} & {\left[\frac { \mathbf { 1 } } { \mathbf { p } } \left(1+\left\|k^{n+1}\right\|_{H^{s}}^{2}+\left\|U^{n}\right\|_{H^{s}}^{2} \mid+\left\|\varepsilon^{n}\right\|_{H^{s}}^{2}+\left\|k^{n}\right\|_{H^{s}}^{2}\right.\right.} \\
& \left.\left.+\frac{1}{k_{\min }^{n}(t)}+\frac{1}{\varepsilon_{\min }^{n}(t)}\right)^{q(s)}+\mathbf{p}\left\|D^{(s+1)} U^{n+1}\right\|_{L^{2}}^{2},\right] \\
\frac{d}{d t}\left\|\varepsilon^{n+1}\right\|_{H^{s}}^{2} \leq C_{s, 1}[ & \frac{\mathbf{1}}{\mathbf{p}}\left(1+\left\|\varepsilon^{n+1}\right\|_{H^{s}}^{2}+\left\|U^{n}\right\|_{H^{s}}^{2} \mid+\left\|\varepsilon^{n}\right\|_{H^{s}}^{2}+\left\|k^{n}\right\|_{H^{s}}^{2}\right. \\
& \left.\left.+\frac{1}{k_{\min }^{n}(t)}+\frac{1}{\operatorname{\varepsilon min}^{n}(t)}\right)^{q(s)}+\mathbf{p}\left\|D^{(s+1)} U^{n+1}\right\|_{L^{2}}^{2}\right] .
\end{aligned}
$$

The strict positivity can be controlled through a maximum principle as was done before if $U^{n}, k^{n}, \varepsilon^{n}, 1 / k^{n}$ and $1 / \varepsilon^{n}$ are bounded on $\left[0, t_{n}\right]$. So we obtain an $H^{s}$ control of the solutions thanks to the three inequalities (3.8)-(3.9)-(3.10) and the maximum principle (the terms of order $s+1$ vanish, using a parameter $p$ small enough).

There exists an integer $r$ and a constant $C_{s, 2}$ both depending on the Sobolev index $s$ such that the quantity $f n$ defined as

$$
f n(t)=\sup _{0 \leq t^{\prime} \leq t}\left\|U^{n}\right\|_{H^{s}}^{2}+\sup _{0 \leq t^{\prime} \leq t}\left\|\varepsilon^{n}\right\|_{H^{s}}^{2}+\sup _{0 \leq t^{\prime} \leq t}\left\|k^{n}\right\|_{H^{s}}^{2}+\frac{1}{k_{\min }^{n}}+\frac{1}{\varepsilon_{\min }^{n}}
$$

obeys to the following equation,

$$
f_{n+1}(t)-f_{n+1}(0) \leq C_{s, 2} t\left(f_{n}(t)+f_{n+1}(t)\right)^{r(s)},
$$


as long as $f_{n}$ and $f_{n+1}$ exist. If $f_{n}$ is bounded on $\left[0, t_{n}\right]$ by $M$, then on $\left[0, \min \left(t_{n}, t_{n+1}\right)\right]$ we get,

$$
f n+1(t) \leq f_{1}(0)+C_{s, 2} t\left(M+f_{n+1}\left(t^{\prime}\right)\right)^{r(s)}
$$

$(f n(0)$ is independent of the iterate number $n)$. Using the same argument as in the a priori estimate one gets that for all $t \leq T=\left(M-f_{1}(0)\right) /\left(C(2 M)^{r(s)}\right), f_{n+1}$ is bounded by $M$. Finally, we ensure that the time of existence does not vanish when $n$ goes to $\infty$ and that the time of existence of the iterates is controlled. Moreover, for all $n$ we have that:

- $U^{n}, k^{n}$ and $\varepsilon^{n}$ belong to $\mathcal{C}\left([0, T] ; H^{s}\left(\mathbb{T}^{3}\right)\right) \cap \mathcal{C}^{1}\left([0, T] ; H^{s-2}\left(\mathbb{T}^{3}\right)\right)$ where $T$ is a time of existence for all iterates,

- they are bounded in $L^{\infty}\left([0, T] ; H^{s}\left(\mathbb{T}^{3}\right)\right)$,

- their (time) derivatives are bounded in $L^{\infty}\left([0, T] ; H^{s-2}\left(\mathbb{T}^{3}\right)\right)$, and

- $k^{n}$ and $\varepsilon^{n}$ are bounded below by a strictly positive given constant.

3.1.3. Passing to the limit. Finally, it can be proven by decreasing the time $\mathrm{T}$, if necessary, that the iterative scheme converges using that, for this time,

$$
\begin{gathered}
\sum_{n \geq 1}\left(\left\|U^{n}-U^{n+1}\right\|_{L^{\infty}\left([0, T] ; L^{2}\right)}^{2}+\left\|\varepsilon^{n}-\varepsilon^{n+1}\right\|_{L^{\infty}\left([0, T] ; L^{2}\right)}^{2}\right. \\
\left.+\left\|k^{n}-k^{n+1}\right\|_{L^{\infty}\left([0, T] ; L^{2}\right)}^{2}\right)<+\infty
\end{gathered}
$$

(see the proof below).

The convergence of the series ensures that $U_{n}, k_{n}$ and $\varepsilon_{n}$ converge in $L^{\infty}\left([0, T] ; L^{2}\right)$ and consequently in $\mathcal{C}\left([0, T] ; L^{2}\right)$ since all the terms are continuous. They also converge in $\mathcal{C}\left([0, T] ; H^{s^{\prime}}\right)$ for $s^{\prime}<s$, since they are bounded in $L^{\infty}\left([0, T] ; H^{s}\right)$ (one can prove this using the Gagliardo-Niremberg inequality — see [18]) Using classical arguments on the regularity of Sobolev spaces (see [18] or [20])) and distribution theory, we see that the limits of the sequences $U_{n}, k_{n}$ and $\varepsilon_{n}$ are solutions of the problem in $\mathcal{C}\left([0, T] ; H^{s}\left(\mathbb{T}^{3}\right)\right) \cap \mathcal{C}^{1}\left([0, T] ; H^{s-2}\left(\mathbb{T}^{3}\right)\right)$ : for instance, since $H^{s^{\prime}}$ and $H^{s^{\prime}-1}$ are algebras for $s^{\prime}$ near enough $s>4+\frac{3}{2}$, in the $k$ equation we get that $U^{n+1} \cdot \nabla k^{n+1}$ converges in $\left.L^{\infty}\left([0, T] ; H^{s^{\prime}-1}\right)\right)$ to $U \cdot \nabla k$ and consequently converges in a distribution sense.

Concerning the pressure term, it can be recovered from the Navier-Stokes equations as usual (see [3] for instance: the series of pressures is also bounded, since $U, k$ and $\varepsilon$ are bounded). Therefore, the theorem is finally proven.

We now prove that the series is indeed convergent:

Lemma 3.1 (Convergence of the series). For $T$ small enough, the infinite sum (3.12) is finite.

Proof. If we define $\left(\alpha_{n}\right)_{n \geq 0}=\left\|U^{n}-U^{n+1}\right\|_{L^{2}}^{2}+\left\|\varepsilon^{n}-\varepsilon^{n+1}\right\|_{L^{2}}^{2}+\left\|k^{n}-k^{n+1}\right\|_{L^{2}}^{2}$, the terms $\left(\alpha_{n}\right)_{n \geq 0}$ verify the following inequality,

$$
\frac{d}{d t} \alpha_{n+1} \leq C\left(\alpha_{n+1}+\alpha_{n}\right)^{r(s)},
$$

with $C$ depending on $s$ and the initial data of $k, U$ and $\varepsilon$ and also depending on the $H^{s}$ bounds of $\left(k^{n}\right)_{n \in \mathbb{N}},\left(\varepsilon^{n}\right)_{n \in \mathbb{N}}$ and $\left(U^{n}\right)_{n \in \mathbb{N}}$, and $r$ strictly positive integer depending 
on $s$ (the inequality is once again obtained using a priori estimates). Since $\left(\alpha_{n}\right)_{n \geq 0}$ is a sequence bounded in $H^{s}$ for instance by a $C^{\prime}$ constant, we obtain

$$
\frac{d}{d t} \alpha_{n+1} \leq C C^{\prime r(s)-1}\left(\alpha_{n+1}+\alpha_{n}\right)
$$

thanks to a Sobolev embedding. Finally, using that $\alpha_{n}(0)=0$, for all $n$ we obtain that

$$
\left(\alpha_{n+1}\right)(t) \leq \int_{0}^{t} \alpha_{n}(s) C C^{\prime r(s)-1} \exp \left(C C^{\prime r(s)-1}(t-s)\right) d s,
$$

and that the sequence

$$
\beta_{n}=\left\|U^{n}-U^{n+1}\right\|_{L^{\infty}\left([0, T] ; L^{2}\right)}^{2}+\left\|\varepsilon^{n}-\varepsilon^{n+1}\right\|_{L^{\infty}\left([0, T] ; L^{2}\right)}^{2}+\left\|k^{n}-k^{n+1}\right\|_{L^{\infty}\left([0, T] ; L^{2}\right)}^{2}
$$

is controlled by

$$
\beta_{n+1} \leq \beta_{n}\left(\exp \left(C C^{\prime r(s)-1} T\right)-1\right) .
$$

Then, for $T$ small enough, $\beta_{n}$ is clearly a convergent series and the lemma is proven. $\square$

3.2. Uniqueness. As the solutions are regular enough (they belong indeed to the functional space $\left.\mathcal{C}\left([0, T] ; H^{2}\left(\mathbb{T}^{3}\right)\right) \cap \mathcal{C}^{1}\left([0, T] ; L^{2}\left(\mathbb{T}^{3}\right)\right)\right)$, the $U \cdot \nabla U$ term does not prevent us from proving uniqueness. So adapting the proofs of uniqueness of parabolic equations (see [22] and Navier-Stokes equations (see [16]) leads to uniqueness.

More precisely, we will just prove an inequality for the Navier-Stokes equation for two solutions of the problem $u$ and $v$ with same initial data. We denote by $k_{u}, \varepsilon_{u}$ (resp. $\left.k_{v}, \varepsilon_{v}\right)$ ) the solutions of the problems associated with $u$ and $v$.

Using classical arguments for the Navier-Stokes equation without a second member $([23]$ ), we get (with $w=u-v)$ :

$$
\frac{d}{d t}\|w\|_{L} \leq C|| w\left\|_{L}\right\| v \|_{L^{4}}^{4}+\int \nabla x \cdot\left(a_{u}\left(\nabla u+\nabla u^{T}\right)\right)-\left(a_{v}\left(\nabla v+\nabla v^{T}\right)\right) \cdot w
$$

and for some $C, a_{u}=\frac{k_{u}}{\varepsilon_{u}}$ and $a_{v}=\frac{k_{v}}{\varepsilon_{v}}$.

First, one gets:

$$
\begin{aligned}
& \int \nabla x \cdot\left(a_{u}\left(\nabla u+\nabla u^{T}\right)\right)-\left(a_{v}\left(\nabla v+\nabla v^{T}\right)\right) \cdot w \\
= & -\frac{1}{2} \int\left(a_{u}\left(\nabla u+\nabla u^{T}\right)\right)-\left(a_{v}\left(\nabla v+\nabla v^{T}\right)\right):\left(\nabla w+\nabla w^{T}\right) .
\end{aligned}
$$

Using some equalities, one gets:

$$
\begin{aligned}
& \left(a_{u}\left(\nabla u+\nabla u^{T}\right)\right)-\left(a_{v}\left(\nabla v+\nabla v^{T}\right)\right):\left(\nabla w+\nabla w^{T}\right) \\
= & -a_{u}\left(\nabla w+\nabla w^{T}\right):\left(\nabla w+\nabla w^{T}\right)+\left(a_{u}-a_{v}\right)\left(\nabla w+\nabla w^{T}\right):\left(\nabla v+\nabla v^{T}\right),
\end{aligned}
$$

so that

$$
\begin{aligned}
& \int \nabla x \cdot\left(a_{u}\left(\nabla u+\nabla u^{T}\right)\right)-\left(a_{v}\left(\nabla v+\nabla v^{T}\right)\right) \cdot w \\
\leq & \frac{1}{2}\left\|\left(\nabla w+\nabla w^{T}\right):\left(\nabla v+\nabla v^{T}\right)\right\|_{L^{\infty}} \int\left|a_{u}-a_{v}\right| \\
\leq & \frac{1}{2} \times 4 \max \left(\|u\|_{H^{s}},\|v\|_{H^{s}}\right)\left\|a_{u}-a_{v}\right\|_{L^{1}} .
\end{aligned}
$$


One can notice that

$$
\begin{aligned}
a_{u}-a_{v} & =\frac{k_{u}}{\varepsilon_{u}}-\frac{k_{v}}{\varepsilon_{v}} \\
& =\frac{k_{u}-k_{v}}{\varepsilon_{u}}+k_{v}\left(\frac{1}{\varepsilon_{u}}-\frac{1}{\varepsilon_{v}}\right) .
\end{aligned}
$$

Using Sobolev embeddings, that $1 / \varepsilon_{u}$ and $1 / \varepsilon_{v}$ are bounded below by a strictly positive constant, that $k_{u}$ and $k_{v}$ are $L^{\infty}$-bounded and some algebra manipulations, one gets that:

$$
\begin{aligned}
\left\|a_{u}-a_{v}\right\|_{L^{1}} \leq & \left(\max _{\mathbb{T}} k_{u}+\max _{\mathbb{T}} k_{v}\right) \times \max _{\mathbb{T}} \frac{1}{\varepsilon_{u}}\left\|k_{u}-k_{v}\right\|_{L^{1}} \\
& +\left(\max _{\mathbb{T}} k_{v}\right)^{2} \max _{\mathbb{T}} \frac{1}{\varepsilon_{u}} \times \max _{\mathbb{T}} \frac{1}{\varepsilon_{v}}\left\|\varepsilon_{u}-\varepsilon_{v}\right\|_{L^{1}} .
\end{aligned}
$$

Using the fact that $L^{1}$ norms are controlled by $L$-norms on the Torus, one gets that there exists a constant $C$ depending on $\max _{\mathbb{T}} k_{u}, \max _{\mathbb{T}} k_{v}, \max _{\mathbb{T}} \frac{1}{\varepsilon_{u}}$ and $\max _{\mathbb{T}} \frac{1}{\varepsilon} v$, such that

$$
\begin{aligned}
& \int \nabla x \cdot\left(a_{u}\left(\nabla u+\nabla u^{T}\right)\right)-\left(a_{v}\left(\nabla v+\nabla v^{T}\right)\right) \cdot w \\
& \leq C\left(\left\|k_{u}-k_{v}\right\|_{L^{2}}+\left\|\varepsilon_{u}-\varepsilon_{v}\right\|_{L^{2}}\right)
\end{aligned}
$$

Finally, there exists some $C$ depending on $\max _{\mathbb{T}} k_{u}, \max _{\mathbb{T}} k_{v}, \max _{\mathbb{T}} \frac{1}{\varepsilon} u$ and $\max _{\mathbb{T}} \frac{1}{\varepsilon} v$ and the $H^{s}$ norms of $k_{u}, k_{v}, \varepsilon_{u}, \varepsilon_{v}$, such that:

$$
\frac{d}{d t}\|w\|_{L} \leq C\left(\|w\|_{L}^{2}+\left\|k_{u}-k_{v}\right\|_{L^{2}}+\left\|\varepsilon_{u}-\varepsilon_{v}\right\|_{L^{2}}\right) .
$$

We would be able to prove the same kind of inequality for $k$ and $\varepsilon$ equations, i.e.,

$$
\begin{aligned}
& \frac{d}{d t}\left\|k_{u}-k_{v}\right\|_{L} \leq C\left(\|w\|_{L}^{2}+\left\|k_{u}-k_{v}\right\|_{L^{2}}+\left\|\varepsilon_{u}-\varepsilon_{v}\right\|_{L^{2}}\right), \\
& \frac{d}{d t}\left\|\varepsilon_{u}-\varepsilon_{v}\right\|_{L} \leq C\left(\|w\|_{L}^{2}+\left\|k_{u}-k_{v}\right\|_{L^{2}}+\left\|\varepsilon_{u}-\varepsilon_{v}\right\|_{L^{2}}\right),
\end{aligned}
$$

which is enough to prove the uniqueness of solutions by summing these three inequalities.

\section{Study of a simplified $k-\varepsilon$ model}

Here we simplify the model by assuming the system is initially at rest so that $U=0$ - as is the case, for instance, in the early development of a Rayleigh-Taylor or Richtmyer-Meshkov mixing layer (see [5]). Consequently, we only take account of the following simplified $k-\varepsilon$ equations:

$$
\begin{aligned}
& \frac{\partial k}{\partial t}-\nabla x \cdot\left(c_{\mu} \frac{k^{2}}{\varepsilon} \nabla k\right)+\varepsilon=0, \quad k(0, x)=k^{0}(x) \in H^{7}\left(\mathbb{T}^{3}\right), \\
& \frac{\partial \varepsilon}{\partial t}-\nabla \cdot\left(c_{\varepsilon} \frac{k^{2}}{\varepsilon} \nabla \varepsilon\right)+c_{2} \frac{\varepsilon^{2}}{k}=0, \quad \varepsilon(0, x)=\varepsilon^{0}(x) \in H^{7}\left(\mathbb{T}^{3}\right) .
\end{aligned}
$$

We make a dimensional analysis which leads us to compute an asymptotic expansion. 
4.1. Dimensional analysis. We analyze the different terms of the equations by making a change of variables:

- $x \hookrightarrow \tilde{x}=\frac{x}{L}$, with $L$ a typical length scale,

- $t \hookrightarrow \tilde{t}=\frac{t}{T}$, with $T$ a typical time scale,

- $\varepsilon \hookrightarrow \tilde{\varepsilon}=\frac{\varepsilon}{\varepsilon^{0}}$, with $\varepsilon^{0}$ a typical rate of dissipation of turbulent energy scale,

- $k \hookrightarrow \tilde{k}=\frac{k}{k^{0}}$, with $k^{0}$ a typical turbulent energy scale.

Thanks to the change of variables, we obtain:

$$
\frac{\partial k}{\partial t}-\eta \nabla \cdot\left(\frac{k^{2}}{\varepsilon} \nabla k\right)+A \varepsilon=0, \frac{\partial \varepsilon}{\partial t}-\eta \nabla \cdot\left(\frac{c_{\varepsilon}}{c_{\mu}} \frac{k^{2}}{\varepsilon} \nabla \varepsilon\right)+c_{2} A \frac{\varepsilon^{2}}{k}=0
$$

where $A=\frac{\varepsilon^{0} T}{k^{0}}$ and $\eta=c_{\mu} \frac{\left(k^{0}\right)^{2} T}{\varepsilon^{0} L^{2}}$ are dimensionless numbers.

For instance, we have the following numerical data (in c.g.s. system,) for RayleighTaylor instabilities in dense hot plasma (see [24]):

$$
\begin{aligned}
& k^{0}=10^{16} \mathrm{~cm}^{2} / \mathrm{s}^{2} \quad T=10^{-3} \mathrm{~s} \quad A=0.5 \\
& \varepsilon^{0}=5.10^{18} \mathrm{~cm}^{2} / \mathrm{s}^{3} \quad L=10^{7} \mathrm{~cm} \quad \eta \sim 10^{-5} \text {. }
\end{aligned}
$$

As can be noticed, $\eta$ is small for the physical applications we study. This is why we expand $\varepsilon$ and $k$ in formal series (see next subsection).

We can also write $\eta$ as $c_{\mu} A\left(\frac{\left(k^{0}\right)^{\frac{3}{2}}}{\varepsilon^{0} L}\right)^{2}$, with $\frac{\left(k^{0}\right)^{\frac{3}{2}}}{\varepsilon^{0}}$ representing the typical length of turbulent vortices (see [17]). So a small $\eta$ is equivalent to neglecting diffusion of vortices because they are too small. Another equivalent approach is to say (writing $\eta$ as $\left.\frac{c_{\mu}}{A} \times\left(T \frac{\sqrt{k^{0}}}{L}\right)^{2}\right)$ that the typical time of creation of the vortices $\frac{L}{\sqrt{k^{0}}}$ is large enough so that vortices cannot diffuse turbulence.

4.2. Bounds for $k$ and $\varepsilon$. We obtain bounds for the solutions of the simplified system which are independent of $\eta$ and which allow us to control nonlinear terms.

Proposition 4.1 (Maximum principle for $k$ and $\varepsilon$ ). Let $k^{0}, \varepsilon^{0}$ belong to $H^{7}\left(\mathbb{T}^{3}\right)$ and be bounded below by a strictly positive constant. Let $k$ and $\varepsilon$ be strictly positive solutions of (1.7) and (1.8) and belong to $C^{1}\left([0, T] ; H^{5}\left(\mathbb{T}^{3}\right)\right)$. Then we get that $\forall x \in \mathbb{T}^{3}$ and $\forall t \in[0, T]$

$$
\begin{gathered}
k(t, x) \leq k_{\max }(0), \\
\varepsilon(t, x) \leq \varepsilon_{\max }(0), \\
k(t, x) \geq k_{\min }(0)-A \varepsilon_{\max }(0) t, \\
\varepsilon(t, x) \geq \varepsilon_{\min }(0) /\left(1-c_{2} \frac{\varepsilon_{\min }(0)}{\varepsilon_{\max }(0)} \log \left(1-\frac{t A \varepsilon_{\max }(0)}{k \min (0)}\right)\right) .
\end{gathered}
$$


Proof. We only prove the result of the lower bound of $\varepsilon$ (we admit the three other results, for which the maximum principle for parabolic equations is more simple to use). Let's introduce $\delta>1$ and the function

$$
r_{\delta}(t, x)=\left(1-c_{2} \frac{\varepsilon_{\min }(0)}{\varepsilon_{\max }(0)} \log \left(1-\frac{t A \varepsilon_{\max }(0)}{k_{\min }(0)}\right)\right) \frac{\delta}{\varepsilon_{\min }(0)}-\frac{1}{\varepsilon(t, x)} .
$$

First one remarks that

$$
r_{\delta}(0, x)=\frac{\delta}{\varepsilon_{\min }(0)}-\frac{1}{\varepsilon(0, x)}>0 \quad \text { since } \quad \delta>1 .
$$

Moreover, $r_{\delta}$ belongs to $\mathcal{C}^{1}\left([0, T], \mathcal{C}^{2}\left(\mathbb{T}^{3}\right)\right)$, since $\varepsilon$ has $C^{1}\left([0, T] ; H^{5}\left(\mathbb{T}^{5}\right)\right)$ regularity. Because $[0, T] \times \mathbb{T}^{3}$ is a compact set, $r \delta$ admits a minimum on $[0, T] \times \mathbb{T}^{3}$; so there exists $x$ and $s$ for which the minimum is reached. This minimum is also a local minimum of $\varepsilon$ in space; as a consequence, $\nabla \varepsilon(s, x)=0$ and $\Delta \varepsilon(s, x) \geq 0\left(\mathbb{T}^{3}\right.$ is an open set).

So the $\varepsilon$ equation at point $(\mathrm{s}, \mathrm{x})$ gives us

$$
\frac{\partial \varepsilon}{\partial t}-\eta \frac{k^{2}}{\varepsilon} \Delta \varepsilon+c_{2} A \frac{\varepsilon^{2}}{k}=0,
$$

and we get, using the strict positivity of $\varepsilon$ and $k$ :

$$
\frac{\partial \varepsilon(s, x)}{\partial t} \geq-c_{2} A \frac{\varepsilon^{2}}{k} \quad \text { and } \quad \frac{\partial 1 / \varepsilon(s, x)}{\partial t} \leq c_{2} A \frac{1}{k} .
$$

Finally,

$$
\begin{aligned}
\frac{\partial r_{\delta}}{\partial t}(s, x) & \geq\left(c_{2} A \frac{\delta}{k_{\min }(0)} /\left(1-\frac{t A \varepsilon_{\max }(0)}{k_{\min }(0)}\right)\right)-c_{2} \frac{A}{k} \\
& \left.>c_{2} A \frac{1}{k_{\min }(0)-t A \varepsilon_{\max }(0)}-c 2 \frac{A}{k} \text { (since } \delta>1\right)
\end{aligned}
$$

$>0$ (using the inequality controlling the lower bound of $k$ ),

so the minimum can only be reached in $s=0$, since if $s>0$, for $s^{\prime}<s$ such $s^{\prime}-s$ is small enough, $r_{\delta}\left(s^{\prime}, x\right)<r_{\delta}(s, x)$.

As a consequence, $r_{\delta}$ is strictly positive for all $t$, and, letting $\delta$ tend to one, one gets the result.

Let us observe that we also obtain a time of strict positivity $T$ independent of $\eta$ whose value is $k_{\min } /\left(A \varepsilon_{\max }\right)$ and for which $k$ and $\varepsilon$ remain positive. Nonetheless, this time is of the same order as of the typical time (see numerical data above).

4.3. Asymptotic analysis of the system. The values used in physics lead us to make an asymptotic series expansion in $\eta$ ( $\eta$ tends to zero) in order to approach the real solution; we write $k$ and $\varepsilon$ as:

$$
k=\sum_{n=0}^{\infty} k_{n} \eta^{n}, \quad \varepsilon=\sum_{n=0}^{\infty} \varepsilon_{n} \eta^{n} .
$$

We first establish properties of the differential systems obtained by expanding in a series expansion in $\eta$. Then the real solution is compared with the truncated series. 
Moreover, we obtain that the more $\eta$ decreases, the more the real solution remains positive. Replacing $k$ and $\varepsilon$ by their expansion we get the following systems. We limit our study to second order even if it can be extended further: although the main result concerns only zeroth and first order systems, we need the second order to prove it.

zeroth order system

$$
\begin{aligned}
& \frac{\partial k_{0}}{\partial t}+A \varepsilon_{0}=0, \quad k_{0}(0, .)=k^{0}(.), \\
& \frac{\partial \varepsilon_{0}}{\partial t}+c_{2} A \frac{\varepsilon_{0}^{2}}{k_{0}}=0, \quad \varepsilon_{0}(0, .)=\varepsilon^{0}(.) .
\end{aligned}
$$

\section{First order system}

$$
\begin{aligned}
& \frac{\partial k_{1}}{\partial t}-\nabla \cdot\left(\frac{k_{0}^{2}}{\varepsilon_{0}} \nabla k_{0}\right)+A \varepsilon_{1}=0, \quad k_{1}(0, .)=0, \\
& \frac{\partial \varepsilon_{1}}{\partial t}-\nabla \cdot\left(\frac{c_{\varepsilon}}{c_{\mu}} \frac{k_{0}^{2}}{\varepsilon_{0}} \nabla \varepsilon_{0}\right)+c_{2} A\left(\frac{2 \varepsilon_{0} \varepsilon_{1}}{k_{0}}-\frac{\varepsilon_{0}^{2} k_{1}}{k_{0}^{2}}\right)=0, \quad \varepsilon_{1}(0, .)=0 .
\end{aligned}
$$

\section{Second order system}

$$
\begin{aligned}
& \frac{\partial k_{2}}{\partial t}-\nabla \cdot\left(\frac{k_{0}^{2}}{\varepsilon_{0}} \nabla k_{1}+\frac{2 k_{0} k_{1}}{\varepsilon_{0}} \nabla k_{0}-\frac{k_{0}^{2} \varepsilon_{1}}{\varepsilon_{0}^{2}} \nabla k_{0}\right)+A \varepsilon_{2}=0, \\
& \frac{\partial \varepsilon_{2}}{\partial t}-\frac{c_{\varepsilon}}{c_{\mu}} \nabla \cdot\left(\frac{k_{0}^{2}}{\varepsilon_{0}} \nabla \varepsilon_{1}+\frac{2 k_{0} k_{1}}{\varepsilon_{0}} \nabla \varepsilon_{0}-\frac{k_{0}^{2} \varepsilon_{1}}{\varepsilon_{0}^{2}} \nabla \varepsilon_{0}\right) \\
& \quad+c_{2} A\left(\frac{2 \varepsilon_{0} \varepsilon_{2}}{k_{0}}-\frac{\varepsilon_{0}^{2} k_{2}}{k_{0}^{2}}-2 \frac{\varepsilon_{0} \varepsilon_{1} k_{1}}{k_{0}^{2}}+\frac{\varepsilon_{0}^{2} k_{1}^{2}}{k_{0}^{3}}+\frac{\varepsilon_{1}^{2}}{k_{0}}\right)=0, \\
& k_{2}(0, .)=0, \quad \varepsilon_{2}(0, .)=0 .
\end{aligned}
$$

After some computations we obtain the following solutions of these systems.

Proposition 4.2 (Solutions of the systems). Let $k^{0}$, $\varepsilon^{0}$ belong to $H^{7}$ and be strictly positive. Define $k_{0}$ and $\varepsilon_{0}$ by:

$$
\begin{aligned}
& k_{0}(t, x)=k^{0}(x)\left(1+\left(c_{2}-1\right) A \frac{\varepsilon^{0}(x)}{k^{0}(x)} t\right)^{\frac{1}{1-c_{2}}}, \\
& \varepsilon_{0}(t, x)=\varepsilon^{0}(x)\left(1+\left(c_{2}-1\right) A \frac{\varepsilon^{0}(x)}{k^{0}(x)} t\right)^{\frac{c_{2}}{1-c_{2}}} ;
\end{aligned}
$$

$k_{0}$ and $\varepsilon_{0}$ are solutions of the zero-order system, belong to $C^{\infty}\left(\left[0, \infty\left[; H^{7}(\mathbb{R})\right)\right.\right.$ and remain strictly positive.

$k_{1}$ and $\varepsilon_{1}$ exist, are unique and belong to $C^{\infty}\left([0, \infty] ; H^{5}(\mathbb{R})\right)$. Moreover their growth and those of their derivatives is at worst polynomial in time.

$k_{2}$ and $\varepsilon_{2}$ exist, are unique and belong to $C^{\infty}\left([0, \infty] ; H^{3}(\mathbb{R})\right)$. Moreover their growth and those of their derivatives is at worst polynomial in time.

Proof. For the zero-order system it is immediate $([11,19])$, by doing the computations, that the given functions are solutions. 
For the first order system, define $B$, the matrix

$$
\mathcal{B}=\left(\begin{array}{cc}
0 & A \\
-c_{2} A \frac{\varepsilon_{0}^{2}}{k_{0}} & c_{2} A \frac{2 \varepsilon_{0}}{k_{0}^{2}}
\end{array}\right), \quad \text { and } \quad v(x)=\left(\begin{array}{c}
k_{1}(x) \\
\varepsilon_{1}(x)
\end{array}\right) .
$$

Using $k_{0}$ and $\varepsilon_{0}$ properties, we get $\mathcal{B} \in C^{\infty}\left([0, \infty] ; H^{7}(\mathbb{R})\right)$.

Besides, $v$ satisfies

$$
\frac{d v(x)}{d t}+\mathcal{B}(t, x) v(x)=f(t, x), \quad v(0, x)=\left(\begin{array}{l}
0 \\
0
\end{array}\right)
$$

( $f$ depends uniquely on $k_{0}$ and $\varepsilon_{0}$ and belongs to $C^{\infty}\left([0, \infty] ; H^{5}(\mathbb{R})\right)$ ). The classical theory of ODE's gives us existence and uniqueness of $k_{1}$ and $\varepsilon_{1}$. Moreover, since the growth of $\mathcal{B}$ and $f$ is at worst polynomial (they both depend on $\varepsilon_{0}$ and $k_{0}$ ), this ensures the growth of $k_{1}, \varepsilon_{1}$, and their derivatives are at worst polynomial.

The proof for the second order system is similar.

4.4. A priori estimates. We now compare $k$ and $\varepsilon$ with their second order expansion with respect to $\eta$ in the $H^{2}$-norm. Let us define

$$
K=k-\sum_{n=0}^{2} \eta^{n} k_{n} \quad \text { and } \quad E=\varepsilon-\sum_{n=0}^{2} \eta^{n} \varepsilon_{n} .
$$

We obtain the following equations for $K$ and $E$ :

$$
\begin{aligned}
& \frac{\partial K}{\partial t}+\eta \nabla \cdot\left(F_{1}\right)+A E=0, K(0, .)=0, \\
& \frac{\partial E}{\partial t}+\frac{c_{\varepsilon}}{c_{\mu}} \eta \nabla \cdot\left(F_{2}\right)+c_{2} A \times\left(F_{3}\right)=0, E(0, .)=0,
\end{aligned}
$$

with:

$$
\begin{array}{r}
\left(F_{1}\right)=\left(\frac{k_{0}^{2}}{\varepsilon_{0}} \nabla k_{0}+\eta\left(\frac{k_{0}^{2}}{\varepsilon_{0}} \nabla k_{1}+\left(\frac{2 k_{0} k_{1}}{\varepsilon_{0}}-\frac{k_{0}^{2} \varepsilon_{1}}{\varepsilon_{0}^{2}}\right) \nabla k_{0}\right)-\frac{k^{2}}{\varepsilon} \nabla k\right), \\
\left(F_{2}\right)=\left(\frac{k_{0}^{2}}{\varepsilon_{0}} \nabla \varepsilon_{0}+\eta\left(\frac{k_{0}^{2}}{\varepsilon_{0}} \nabla \varepsilon_{1}+\frac{2 k_{0} k_{1}}{\varepsilon_{0}} \nabla \varepsilon_{0}-\frac{k_{0}^{2} \varepsilon_{1}}{\varepsilon_{0}^{2}} \nabla \varepsilon_{0}\right)-\frac{k^{2}}{\varepsilon} \nabla \varepsilon\right), \\
\left(F_{3}\right)=\left(\frac{\varepsilon^{2}}{k}-\frac{\varepsilon_{0}^{2}}{k_{0}}+\eta\left(\frac{\varepsilon_{0}^{2} k_{1}}{k_{0}^{2}}-\frac{2 \varepsilon_{0} \varepsilon_{1}}{k_{0}}\right)\right. \\
\left.+\eta^{2}\left(\frac{\varepsilon_{0}^{2} k_{2}}{k_{0}^{2}}-\frac{\varepsilon_{1}^{2}}{k_{0}}-\frac{2 \varepsilon_{0} \varepsilon_{2}}{k_{0}}+2 \frac{\varepsilon_{0} \varepsilon_{1} k_{1}}{k_{0}^{2}}-\frac{\varepsilon_{0}^{2} k_{1}^{2}}{k_{0}^{3}}\right)\right) .
\end{array}
$$

We define $T \mapsto B(T)$ by

$$
B(T)^{-1}=\inf _{0 \leq t \leq T, x \in \mathbb{T}^{3}}\left(k(t, x), k_{0}(t, x), \varepsilon(t, x), \varepsilon_{0}(t, x)\right),
$$

which is bounded, since $k$ and $\varepsilon$ are bounded and strictly positive on $[0, T]$.

We assess the norm of the different terms to obtain energy estimates for the whole system. To simplify computations, we suppose $\eta<1$ ( $\eta$ tends to zero..). We get: 
Lemma 4.1. Let $k^{0}, \varepsilon^{0}$ belong to $H^{7}$ and be strictly positive. Let $k$ and $\varepsilon$ be strictly positive solutions of (1.7) and (1.8) and belong to $C^{1}\left([0, T] ; H^{5}\left(\mathbb{T}^{3}\right)\right)$. There exists a positive function $f$ depending in a polynomial way on time ${ }^{2}$ but independent of $\eta$ such that:

$$
\begin{aligned}
\left|\left(F_{1}\right)(t, x)\right|+|(F 2)(t, x)| \leq & B(T)^{5} f(T)\left[\left(\|K\|_{H^{2}}+\|E\|_{H^{2}}\right)^{6}+\eta^{2}\right], \\
\left|D^{1}\left(F_{1}\right)(t, x)\right|+\left|D^{1}\left(F_{2}\right)(t, x)\right| \leq & B(T)^{5} f(T)\left[\eta^{2}\right. \\
& +\left(1+\left|D^{2} K(t, x)\right|+\left|D^{2} E(t, x)\right|\right) \\
& \left.\times\left(\|K\|_{H^{2}}+\|E\|_{H^{2}}\right)^{7}\right], \\
\left|\left(F_{3}\right)(t, x)\right|+\left|D^{1}\left(F_{3}\right)(t, x)\right| \leq & B(T)^{5} f(T)\left[\left(\|K\|_{H^{2}}+\|E\|_{H^{2}}\right)^{6}+\eta^{3}\right], \\
\left|D^{2}\left(F_{3}\right)(t, x)\right| \leq & B(T)^{5} f(T)\left[\eta^{3}\right. \\
& +\left(1+\left|D^{2} K\right|(t, x)+\left|D^{2} E(t, x)\right|\right) \\
& \left.\times\left(\|K\|_{H^{2}}+\|E\|_{H^{2}}\right)^{7}\right] .
\end{aligned}
$$

Proof. We prove the result for $\left|\left(F_{3}\right)\right|$. Using the mean value theorem we get,

$$
\frac{1}{k}=\frac{1}{k_{0}}-\frac{k-k_{0}}{k_{0}^{2}}+\frac{\left(k-k_{0}\right)^{2}}{k_{0}(t, x)^{3}}+c(t, x)\left(k-k_{0}\right)^{3} \text { with }|c(t, x)| \leq B(T)^{3} .
$$

We put this formula in $\left(F_{3}\right)$ and replace $k$ by $K+k_{0}+\eta k_{1}+\eta^{2} k_{2}, \varepsilon$ by $E+\varepsilon_{0}+\eta \varepsilon_{1}+$ $\eta^{2} \varepsilon_{2}$. All zero, first and second order terms independent of $K$ and $E$ vanish. The remaining terms which do not depend on $K$ and $E$ are preceded by $\eta^{3}$ at least and their growth in time is at worst polynomial thanks to results obtained on $k_{0}, k_{1}, k_{2}, \varepsilon_{0}, \varepsilon_{1}, \varepsilon_{2}$ (it gives us the bound $\eta^{3} B(T)^{6} f(T)$ ).

Also, $E^{2}, E, E^{2} K^{3}, E K^{3}, K^{3}, E^{2} K^{2}, E K^{2}, K^{2}, E^{2} K, E K$ and $K$ terms appear: these terms are multiplied by functions depending on $k_{0}-\varepsilon_{2}$ and $\eta$ which can be majored independently of $\eta$ as $\eta \leq 1$ and whose growth is at worst polynomial, thanks to results obtained on $k_{0}, \ldots, \varepsilon_{2}$ (it gives us the other bound: $\left.B(T)^{5} f(T)\left[\left(\|K\|_{H^{2}}+\|E\|_{H^{2}}\right)^{6}\right]\right)$.

So we get the result; we use the same method for the others results.

Proposition 4.3 ( $H^{2}$ estimates). Let $k^{0}$, $\varepsilon^{0}$ belong to $H^{7}$ and be strictly positive. Let $k$ and $\varepsilon$ be strictly positive solutions of (1.7) and (1.8) and belong to $C^{1}\left([0, T] ; H^{5}\left(\mathbb{T}^{3}\right)\right)$. There exists an $f$ depending in a polynomial way of time but independent of $\eta$ such that:

$$
\begin{array}{r}
\frac{d}{d t}\left\|D^{2} K\right\|_{L^{2}}^{2} \leq B(T)^{5} f(t)\left[\left(\|K\|_{H^{2}}^{2}+\|E\|_{H^{2}}^{2}\right)^{9}+\eta^{3}\right], \\
\frac{d}{d t}\left\|D^{2} E\right\|_{L^{2}}^{2} \leq B(T)^{5} f(t)\left[\left(\|K\|_{H^{2}}^{2}+\|E\|_{H^{2}}^{2}\right)^{9}+\eta^{3}\right], \\
\frac{d}{d t}\left(\|K\|_{H^{2}}^{2}+\|E\|_{H^{2}}^{2}\right) \leq B(T)^{5} f(t)\left[\left(\|K\|_{H^{2}}^{2}+\|E\|_{H^{2}}^{2}\right)^{9}+\eta^{3}\right] .
\end{array}
$$

\footnotetext{
${ }^{2}$ by this we mean $f \leq C(1+t)^{n}$ for some integer $n$
} 
Proof. First, one can easily note that using equations (4.3) and (4.4), one gets:

$$
\begin{gathered}
\left.\frac{d}{d t}|| K\right|_{L^{2}} ^{2} \leq|\nabla(F 1)| \int|K|+A \int|K E| \\
\left.\frac{d}{d t}|| E\right|_{L^{2}} ^{2} \leq|\nabla(F 2)| \int|K|+A \int|E| \times|(F 3)| \\
\frac{d}{d t}\left\|D^{1} K\right\|_{L^{2}}^{2} \leq|\nabla(F 1)| \int|\Delta K|+A \int|\nabla K \cdot \nabla E|, \\
\frac{d}{d t}|| D^{1} E \|_{L^{2}}^{2} \leq\left(\frac{c_{\varepsilon}}{c_{\mu}}|\nabla(F 2)| \int|\Delta E|+c_{2} A|\nabla(F 3)| \int|\nabla E|\right) .
\end{gathered}
$$

Thanks to the bounds on (F1), (F2) and (F3) and their derivatives, as soon as you are able to prove (4.5) and (4.6) you obtain inequality (4.7).

We prove only the result in the $L^{2}$-norm of $D^{2} K$ (inequality (4.5)), since the other proofs are very similar. We differentiate two times in $\alpha \in \mathbb{N}^{3}(|\alpha|=2)$ the $K$ equation and we multiply it by $\partial^{\alpha} K$. Integrating by parts the second term, one gets:

$$
\frac{d}{d t} \int\left|\partial^{\alpha} K\right|^{2}=-\eta \int\left(\partial^{\alpha}(F 1)\right) \cdot \partial^{\alpha} \nabla K-A \int \partial^{\alpha} K \partial^{\alpha} E .
$$

Studying precisely $\partial^{\alpha}(F 1)$, terms in $K$ of third order of order are those coming from $\partial^{\alpha}\left(\frac{k^{2}}{\varepsilon} \nabla k\right)$ and especially $\frac{k^{2}}{\varepsilon} \partial^{\alpha} \nabla k$.

Using again the mean value theorem on $1 / \varepsilon, 1 / \varepsilon^{2}, 1 / \varepsilon^{3}$, we obtain that the terms of $\partial^{\alpha} F 1$ include $k^{2} / \varepsilon\left(\partial^{\alpha} \nabla K\right)$, linear terms in $\Delta K$ and $\Delta E$ (these functions are only multiplied by first derivatives of $E$ and $K, E$ and $K$ and independent functions of $\eta$ ), other terms in $\nabla K, \nabla E, K$ and $E$ and finally terms in $\eta^{2} f(t) \quad(f(t)$ can be bounded independently of $E, K$ and $\eta$ thanks to bounds on terms $\left.k_{0}, k_{1}, k_{2}, \varepsilon_{0}, \varepsilon_{1}, \varepsilon_{2}\right)$.

Making use of the diffusive term $k^{2} / \varepsilon\left(\partial^{\alpha} \nabla K\right)$ to control all terms of strictly inferior order via Sobolev embeddings, we get the result on $\partial \alpha K$. This proof holds for all second derivatives, so for the $H^{2}$-estimate is obtained. Now we are able to prove Theorem 1.2.

Proof. 1.2 Define $S(t)=\|K\|_{H^{2}}^{2}(t)+\|E\|_{H^{2}}^{2}(t)$. Note that $S(0)=0$. Thanks to Proposition 4.3 , there exists $f$ a function of time bounded by $C(1+t)^{n}$ such that we have (we recall that we have already supposed that $\eta \leq 1$ ):

$$
\begin{aligned}
S^{\prime}(t) & \leq B(T)^{5} f(t)\left[S(t)^{9}+\eta^{3}\right] \\
& \leq H(T)\left[S(t)^{9}+\eta^{3}\right] \\
& \leq H(T)\left[S(t)^{9}+1\right],
\end{aligned}
$$

with $H(T)=B(T)^{5} \sup _{0 \leq t \leq T} f(t)$.

Let $T_{1}$ be the first time such that $S\left(T_{1}\right)=1\left(T_{1}\right.$ is independent of $\eta$ thanks to inequality 4.8). For all $t \leq T_{1}, S^{9}(t) \leq S(t)$ and $S^{\prime}(t) \leq H(T)\left[S(t)+\eta^{3}\right]$, so

$$
\forall t \leq T_{1}, S(t) \leq \eta^{3}(\exp (H(T) t)-1) \quad \text { (using Gronwall lemma) }
$$


So we get:

$$
\begin{aligned}
& \forall t \leq T_{1},\|K\|_{H^{2}}(t) \leq \sqrt{\exp (H(T) t)-1} \eta^{\frac{3}{2}} \\
& \forall t \leq T_{1},\|E\|_{H^{2}}(t) \leq \sqrt{\exp (H(T) t)-1} \eta^{\frac{3}{2}}
\end{aligned}
$$

Then using Sobolev embeddings, since $H^{2}\left(\mathbb{T}^{3}\right) \hookrightarrow L^{\infty}\left(\mathbb{T}^{3}\right)$, one gets:

$$
\begin{aligned}
& \forall t \leq T_{1},\left\|k-k_{0}-\eta k_{1}-\eta^{2} k_{2}\right\| \infty(t) \leq C \sqrt{\exp (H(T) t)-1} \eta^{\frac{3}{2}} \leq C \eta^{\frac{3}{2}}, \\
& \forall t \leq T_{1},\left\|\varepsilon-\varepsilon_{0}-\eta \varepsilon_{1}-\eta^{2} \varepsilon_{2}\right\| \infty(t) \leq C \sqrt{\exp (H(T) t)-1} \eta^{\frac{3}{2}} \leq C \eta^{\frac{3}{2}}
\end{aligned}
$$

Using the fact that $k_{2}$ and $\varepsilon_{2}$ are bounded by polynomial functions of time, which are bounded on $[0, T]$ (see Proposition 4.2), one gets the theorem.

From this theorem we obtain the following result:

COROLlary 4.4. The theorem holds for $T_{1}=T$ if $\eta$ is small enough.

Proof. $\quad$ In order to prove the corollary, using inequality (4.9) at time $T_{1}$ one gets:

$$
1 \leq \eta^{3}\left(\exp \left(H(T) T_{1}\right)-1\right)
$$

Thus:

$$
T_{1} \geq \frac{1}{H(T)} \ln \left(1+\frac{1}{\eta^{3}}\right) .
$$

Hence when $\eta$ is small enough, $T_{1}=T$. This ends the proof of the corollary.

\section{REFERENCES}

[1] D. Arnett, J. Bahcall, R. Kirschner and S. Woosley, Supernova 1987a, Ann. Rev. Astron. Astrophys., 27, 629, 1989.

[2] H. Brezis, Analyse Fonctionnelle, Masson, 1983.

[3] P. Constantin and C. Foias, Navier-Stokes Equations, The University of Chicago Press, 1988.

[4] J. Cousteix, Turbulence et couche limite, Cepadues, Septembre, 1989.

[5] S.B. Dalziel, P.F. Linden and D.L. Youngs, Self-similarity and internal structure of turbulence induced by Rayleigh-Taylor instability, J. Fluid Mech., 399, 1-48, 1999.

[6] C. Garcia Vazquez and F. Ortegon Gallego, Sur un problme elliptique non linaire avec diffusion singulire et second membre dans l1, C.r. Acad. sci., 332, 145-150, 2001.

[7] E. Guyon, J.P. Hulin and L. Petit, Hydrodynamique Physique, Intereditions, Editions du C.N.R.S., 1991.

[8] R.I. Klein, C.F. McKee and P. Colella, In the evolution of interstellar medium, L. Blitz, Ed., Astronomical Society of the Pacific Conference Series, 12, 117, 1990.

[9] R.I. Klein, C.F. McKee and P. Colella, The hydrodynamic interaction of shock waves with interstellar clouds. I. Nonradiative shocks in small clouds, Ap. J., 420, 213, 1994.

[10] T. Kumagai, T. Shigeyama, K. Nomoto, M. Itoh, J. Nishimura and S. Tsurata, Gamma-rays, $x$-rays and optical light from the cobalt and the neutron star in sn 1987a, Ap. J., 345, 412-422, 1989.

[11] S. Lasserre, Contribution à l'étude Mathématique et Numérique des Solutions à Support Compact Pour Les Modèles de Turbulence Compressible, PhD thesis, University of Paris VI, 2005.

[12] B. Launder and D. Spalding, Mathematical Models of Turbulence, Academic press, 1972.

[13] J. Lederer and R. Lewandowski, A RANS 3d model with unbounded eddy viscosities, Ann. Inst. Henri Poincaré, 24, 413-441, 2007.

[14] R. Lewandowski, Modèles de turbulence et équations paraboliques, C.R. Acad. Sci. Paris, 317, 835-840, 1993. 
[15] R. Lewandowski and B. Mohammadi, Existence and positivity results for the $\phi-\theta$ and a modified $k-\epsilon$ two-equation turbulence models, Math. Models Methods Appl. Sci., 3, 195-215, 1993.

[16] P.L. Lions, Mathematical Topics in Fluid Mechanics. Vol. 1: Incompressible Models, Clarendon Press, 1996.

[17] A. Llor and P. Bailly, A new turbulent two-field concept for modeling Rayleigh-Taylor, Richtmyer-Meshkov, and Kelvin-Helmholtz mixing layers, Laser and particle beams, 21-3, 305-315, 2003.

[18] A. Majda, Compressible Fluid Flow and Systems of Conservation Laws in Several Space Variables, Springer-Verlag, 1984.

[19] B. Mohammadi and O. Pironneau, Analysis of the $k-\varepsilon$ Turbulence Model, Masson, 1994.

[20] D. Serre, Systèmes de lois de conservation I, Diderot, 1996.

[21] M. Taylor, Partial Differential Equations, Basic Theory, Springer, 1996.

[22] M. Taylor, Partial Differential Equations III, non Linear Equations, Springer, 1996.

[23] T. Temam, Navier-Stokes Equation, Elsevier Science Publisher, 1984.

[24] F. Thais, J. Chize, P. Baclet, A. Benuzzi, S.Bouquet, C. Cherfils, F. Mucchielli, L. Pols, C. Reverdin, R. Teyssier, J. Thbault and P. Troussel, The Astrolab experiment: RayleighTaylor instabilities in supernova, Elsevier, 2000. 